\title{
The effect of object shape and mode of presentation on judgments of apparent volume
}

\author{
BRUCE J. FRAYMAN \\ Aquinas College, Grand Rapids, Michigan 49506 \\ and \\ WILLIAM E. DAWSON \\ University of Notre Dame, Notre Dame, Indiana 46556
}

\begin{abstract}
Apparent volume for geometric solids and life-sized two dimensional representations of these solids was scaled by magnitude estimation. Data were adequately fit by power functions whose exponents were less than those previously reported for judgments of apparent length and area. Object shape and mode of presentation affected both magnitude estimations of apparent volume and the best fitting power function exponents. The influence of shape, both across object shape classes and within the cylinder shape class, appears to depend upon the relative elongation of the vertical dimension.
\end{abstract}

In 1961, Ekman and Junge reported that judgments of apparent length, area, and volume are all proportional to actual physical size, since power function exponents for lines, squares, and solid cubes, determined by ratio estimations, were $1.11, .92$, and 1.01 , respectively. Observers also judged the apparent size of the cubes represented by life-sized perspective drawings, the result being a power function exponent of .79. Since the exponent for the apparent size of the cubes themselves was 1.01, Ekman and Junge concluded that two-dimensional representations (a change in mode of presentation) do not provide sufficient depth cues for adequate perception of apparent volume.

However, other data suggest that exponents for apparent area and apparent volume may be less than 1.0. Teghtsoonian $(1959,1965)$ reported exponents for apparent area that ranged mostly between .76 and .81 for a variety of shapes. She also instructed observers to make magnitude estimations of solid cubes and octahedrons, and obtained exponents ranging between .65 and .74 . Teghtsoonian did not report a significant effect of object shape upon these exponents. She did conclude that the exponent for apparent volume is less than 1.0 and suggested there may be a systematic decrease in exponent as judgments of apparent size shift from length to area to volume, a contention supported by the exponents she measured.

Baird (1970) reviewed these and other studies of the scaling of volume (see Table 1). In Table 1, the median exponent for three-dimensional solids is .74 and that for their two-dimensional representation, .68. Note that Ekman and Junge's exponent of 1.01 for solids is an outrider in an otherwise tight distribution of values, and that the effect of mode of presentation reported by these authors depends upon comparisons with this value. More recently, Moyer, Bradley, Sorenson, Whiting, and Mansfield (1978) have reported power function exponents less than 1.0 for both remembered and perceived size of one-, two-, and three-dimensional objects.

A glance at Table 1 reveals that comparisons across experiments are confounded by differences in methods, object shapes, and mode of presentation. The present paper reports investigations of the effect of shape and mode of presentation on judgments of apparent volume with a single standardized procedure. These studies sought to answer three questions. First, is the exponent for apparent volume around $\mathbf{1 . 0}$ or is it les than the exponents for apparent length and area? Second, does the shape of the object that is judged influence exponents and magnitude estimations for apparent volume? Third, are the results of apparent volume judgments obtained with two-dimensional representations of solids equivalent to those obtained with solid objects?

\section{EXPERIMENT 1}

Subjects were asked to judge the apparent volume of solid objects and life-sized, two-dimensional representations of these objects.

\footnotetext{
Method

Subjects. Twenty volunteer undergraduate students from introductory psychology classes at the University of Notre Dame were subjects. Most had had no prior experience with magnitude estimation, and none had had experience with the judgment of apparent volume. The group consisted of 16 males and 4 females, each of whom received class credit for participation.

Stimull and Apparates. Seven geometric shapes, the Platonic solids of classical geometry (Fejas Toth, 1964)-cubes, tetrahedrons, octahedrons, dodecahedrons, and icosahedrons-plus spheres and cylinders were used. Construction materials were
} 
Table 1

Summary of Previous Volume Scaling Reports (Adapted from Baird, 1970)

\begin{tabular}{|c|c|c|c|c|c|c|}
\hline \multirow[b]{2}{*}{ Source } & \multirow[b]{2}{*}{ Me thod } & \multicolumn{3}{|c|}{ Solid } & \multicolumn{2}{|c|}{ Drawing } \\
\hline & & Cube & Sphere & Octahedron & Cube & Sphere \\
\hline Ekman \& Junge (1961) & RE & 1.01 & & & .79 & \\
\hline Sjöberg (1960) (See Ekman \& Junge, 1961) & ME & .74 & .77 & & & \\
\hline Ekman, Lindman, \& William-Olsson (1961) & $\mathrm{ME}$ & & & & $.71, .68, .59$ & $.69, .66$ \\
\hline Teghtsoonian (1965) & ME & $.72, .67$ & & $.74, .65$ & & \\
\hline
\end{tabular}

Note $-R E=$ ratio estimation; $M E=$ magnitude estimation.

Styrofoam for spheres, wood for cylinders, and cardboard for other shapes, all finished in a uniform shade of white. There were nine solids of each shape, whose volumes increased in the geometric series $8,16,32,64,128,256,512,1,024$, and $2,048 \mathrm{~cm}^{3}$. The cylinders had a constant height-to-width ratio of $2: 1$ across all volumes. All 63 solids were constructed to within $5 \%$ of nominal volume.

Photographs were taken of each solid object from a fixed distance and angle of view that matched those from which the solid objects would be observed. Each final print was enlarged to give a life-sized image of the solids from the viewing distance. Perspective drawings were made by inking line tracings of all contours visible in the photographs. These drawings were not shaded, and they completed the set of 189 stimuli.

In the experimental room, each subject was seated at the table, with a black curtained wall in his line of view. A chinrest located the subject's eyes $45 \mathrm{~cm}$ above the table and $113 \mathrm{~cm}$ from the stimulus. Lighting from twin banks of ceiling-mounted fluorescent lamps minimized shadows of objects placed upon the table. The experimenter stood to the subject's left, midway down the table where stimuli could be easily changed. All stimuli were stored out of the subject's sight.

Procedure. Subjects were run one at a time after a familiarization task in which they gave magnitude estimations of apparent length of lines. Next, they judged the apparent volume of each of the stimuli. They were asked to judge how big the objects appeared to be. Instructions for all experiments were patterned after those of Teghtsoonian (1965). If subjects questioned the concept of apparent size, the experimenter said, "How big the object looks to you" or "How big it seems to be." When presented with a twodimensional representation of a real object, they were instructed to judge the apparent volume of the solid represented.

To prevent fatigue, the 189 judgments were divided among five sessions, generally scheduled every third day. No subject had more than one session on any one day.

At the beginning of each session, the standard $128-\mathrm{cm}^{3}$ sphere, called "10," was presented. Each of the 189 stimuli, including this standard, was presented singly in random orders unique to each subject. Each stimulus was judged once by each subject, who proceeded at his own pace.

\section{Results}

Geometric means for magnitude estimations were calculated for each of the 189 stimuli at each volume. Based upon a least squares criterion, best fitting power function $s^{1}$ were derived relating average estimations to volumes for each of the $\mathbf{2 1}$ mode-shape combinations. Table 2 lists the exponents of these group data power functions. They are all less than 1.0. Generally, solids gave the highest exponent, followed by photographs, and then perspective drawings. Analyses of variance were conducted both upon the individual subject's exponents and upon the magnitude estimations.

For exponents, the main effects of both shape
$[F(6,114)=4.48]$ and mode $[F(2,38)=19.99]$ were significant at the $\mathrm{p}<.001$ level, but the Mode by Shape interaction $[F(12,228)=1.33]$ was not. To pinpoint the sources of these shape and mode effects, pairwise comparisons were made on the marginal means of the exponents of Table 2 in terms of critical mean differences with a Tukey correction to control for Type I errors (Keppel, 1973, pp. 133-144, 305). All comparisons were evaluated at an alpha level of .01. The exponent for cylinders is significantly larger than those for cubes and icosahedrons $\left(C R_{T}=.36\right.$ needed for $\mathrm{p}<.01$ ), but all other comparisons by shape showed nonsignificant differences. ${ }^{2}$ For modes $\left(\mathrm{CR}_{\mathrm{T}}=.023\right.$, $\mathrm{p}<.01$ ), the exponent for solids is significantly larger than those for photographs and perspective drawings, which are not different from each other. Figure 1 shows these mode differences for all shapes combined.

For magnitude estimations, which were converted to $\log$ values for analysis (Stevens \& Guirao, 1962), the main effects of shape $[\mathrm{F}(6,114)=50.46]$, mode $[F(2,38)=20.21]$, and volume $[F(8,152)=697.51]$ were all significant $(\mathrm{p}<.001)$. First-order interactions of Shape by Mode $[F(12,228)=2.71]$, Shape by Volume $[F(48,912)=2.67]$, and Mode by Volume $[F(16,304)=5.52]$ were also significant at the .01 , .001 , and .001 levels, respectively. The second-order interaction was not significant $[F(96,1824)=1.12]$. It should be noted that the Shape by Mode interaction, to be reported later for Experiment 2, was not significant.

Pairwise comparisons were made on log mean magnitude estimations by the method of critical mean differences with a Tukey correction $(\alpha=.01)$. The Mode by Volume interaction (see Figure 1), which is consistent with the significant effect of mode in the analysis of variance of exponents, depends on significant differences between the solids and twodimensional stimuli only at volumes below the $128-\mathrm{cm}^{3}$ standard $\left(\mathrm{CR}_{\mathbf{T}}=.037, \mathrm{p}<.01\right)$. There are no significant differences between the photographs and drawings at any volume. The sources of the Shape by Volume interaction, whose existence is consistent with the significant effect of shape in the analysis of variance of exponents, are difficult to identify, and simple conclusions based upon pairwise comparisons are elusive.

Table 3 shows the mean magnitude estimations for 
Table 2

Exponents of Best-Fitting Power Functions for Experiments 1 and 2

\begin{tabular}{|c|c|c|c|c|c|c|c|c|}
\hline \multirow[b]{3}{*}{ Shape } & \multicolumn{8}{|c|}{ Experiment } \\
\hline & \multicolumn{2}{|c|}{ Solid Mode } & \multicolumn{2}{|c|}{ Photo Mode } & \multicolumn{2}{|c|}{ Drawing Mode } & \multicolumn{2}{|c|}{ Mean } \\
\hline & 1 & 2 & 1 & 2 & 1 & 2 & 1 & 2 \\
\hline Cube & .560 & .600 & .560 & .510 & .550 & .530 & .555 & .545 \\
\hline Tetrahedron & .580 & .610 & .560 & .520 & .560 & .540 & .568 & .553 \\
\hline Icosahedron & .580 & .600 & .550 & .490 & .520 & .500 & .550 & .532 \\
\hline Octahedron & .590 & .600 & .570 & .480 & .540 & .480 & .563 & .518 \\
\hline Dodecahedron & .600 & .630 & .570 & .510 & .550 & .520 & .572 & .554 \\
\hline Sphere & .610 & .580 & .560 & .470 & .550 & .480 & .573 & .512 \\
\hline Cylinder & .610 & .610 & .600 & .510 & .580 & .550 & .596 & .556 \\
\hline Mean & .591 & .604 & .567 & .497 & .547 & .514 & & \\
\hline
\end{tabular}

data collapsed over modes and volumes. In general, tetrahedrons receive the highest means, and spheres and cubes the lowest, with the other shapes intermediate.

Although difficult to represent here, each shape

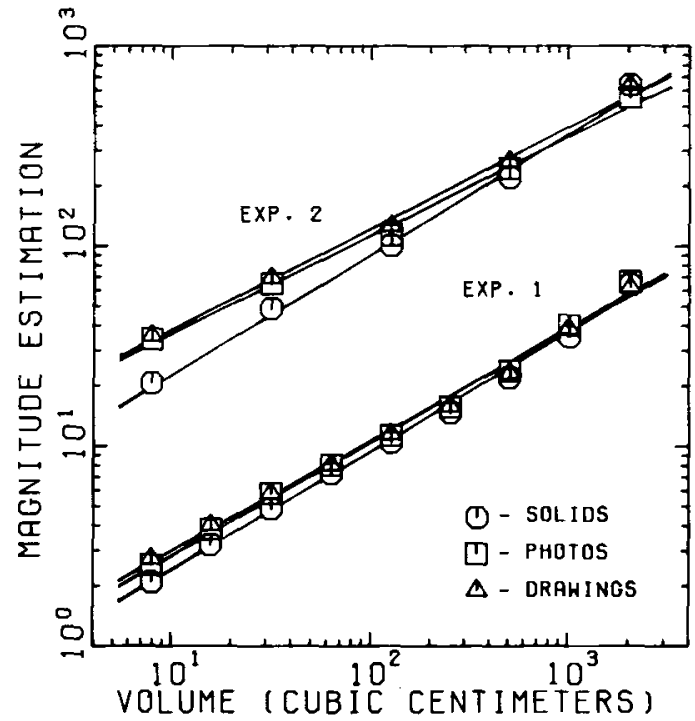

Figure 1. Geometric means of magnitude estimations from Experiments 1 and 2 for solids (octagons), photographs (squares), and drawings (triangles). Note that values for Experiment 2 have been displaced upward $1 \log$ unit. maintains for the most part the same magnitude estimation rank as volume increases, just as the previous comparison of exponents suggests they should. The cylinders are the exception. These increase in rank as volume increases, creating the significant difference in exponent for this shape when compared with cubes and icosahedrons, and contributing to the Shape by Volume interaction in the analysis of magnitude estimations.

This first study leads to the following conclusions: (1) Some shapes (tetrahedrons, and cylinders at some volumes) appear larger than others at equal volumes, and some shapes (spheres, cubes) appear smaller; (2) generally, the same exponent governs the growth of apparent volume for all the shapes used, with the exception of the cylinders, which have a slightly higher exponent, as previously explained; and (3) solids exhibit a slightly higher exponent for apparent volume than do two-dimensional representations, and they appear smaller than their representations at lower volumes.

\section{EXPERIMENT 2}

The repeated measures design in Experiment 1 could have allowed carryover effects to contribute to the results, particularly for judgments across modes. For example, a subject's exposure to solids might thereafter influence judgments of their two-dimensional repre-

Table 3

Mean Magnitude Estimates for Seven Shapes

\begin{tabular}{|c|c|c|c|c|c|c|c|}
\hline Experiment $1 *$ & $\stackrel{S}{S}$ & $\begin{array}{c}\mathrm{Cu} \\
10.67\end{array}$ & $\begin{array}{c}\mathrm{D} \\
11.53 \\
\end{array}$ & $\begin{array}{c}0 \\
11.64 \\
\end{array}$ & $\begin{array}{c}1 \\
11.78 \\
\end{array}$ & $\begin{array}{c}\text { Cy } \\
12.68\end{array}$ & $\underset{14.29}{T}$ \\
\hline Experiment $2 \dagger$ & $\underset{10.52}{S}$ & $\begin{array}{c}\mathrm{Cu} \\
12.02\end{array}$ & $\begin{array}{c}0 \\
12.19\end{array}$ & $\underset{12.30}{I}$ & $\begin{array}{c}\mathrm{D} \\
12.33\end{array}$ & $\begin{array}{c}\text { Cy } \\
13.15\end{array}$ & $\begin{array}{c}T \\
14.66\end{array}$ \\
\hline
\end{tabular}

Note-Underlining indicates nonsignificant difference(s) among included values. To assess any one mean, drop vertically below it to the underlining. Then proceed right or left until encountering nonunderlined means. If such a mean shares no common underlining with the original mean, it is significantly different from it. If a mean has no underlining, it is significantly different from all other means. (See Keppel, 1973.) *For means of $\log$ means, $C R_{T}=.034(p<.01)$. †For means of log means, $C R_{T}=.017(p<.01)$. Convert table entries to common logs when comparing with Tukey critical range values $\left(C R_{T}\right)$. $S=$ sphere, $C u=c u b e, D=d o d e c a h e d r o n, O=O c t a-$ hedron, $I=$ Icosahedron, $C y=$ cylinder, and $T=$ tetrahedron. 
sentations. Therefore, in Experiment 2, mode was a between-groups factor, and the mode of the standard was changed with the mode of the stimuli judged, so there would be no opportunity for familiarization with solids in the experiment.

\section{Method}

Subjects. Sixty new undergraduate subjects, 41 males and $19 \mathrm{fe}-$ males, were randomly divided into three equal groups, one for each mode. Most were unfamiliar with magnitude estimation, and none had prior experience with judgments of apparent volume. Grade credit was given for participation.

Apparatus. The stimuli for Experiment 1 were used, except that the seven geometric shapes in each mode included only the five volumes of $8,32,128,512$, and $2,048 \mathrm{~cm}^{3}$, covering the same volume range as before. Since mode was a between-groups factor, the number of judgments required of each subject dropped from 189 to a more manageable 35 . Stimuli were viewed by subjects under the same conditions as in Experiment 1.

Procedure. The procedure was unchanged, except that a given subject judged objects in only one mode and completed all judgments in a single session. The standard was the same $128-\mathrm{cm}^{3}$ sphere used before, but its mode was changed to match the mode of the objects judged.

\section{Results}

Using geometric means, best fitting power functions were derived for each shape class at each mode. Table 2 lists the exponents, and, as in Experiment 1, all exponents are less than 1.0. Once again, the solids clearly exhibit the highest exponents, with the exponents of the photographs and drawings being lower and nearly identical.

The individual exponents for subjects in the various shape and mode conditions were examined in an analysis of variance. Both shape $[F(6,342)=4.72]$ and mode $[F(2,57)=8.80]$ were significant $(p<.001)$. There was no significant Mode by Shape interaction $[\mathrm{F}(12,342)<1]$.

Pairwise comparisons were conducted on marginal means of exponents in Table 2. For shapes, the exponent for spheres is significantly different from those for cylinders, tetrahedrons, and dodecahedrons $\left(\mathrm{CR}_{\mathrm{T}}=.040, \mathrm{p}<.01\right)$. Other shape comparisons were nonsignificant. The same trends were noted in Experiment 1 . For modes, solids were significantly different from photographs and drawings, but there was no difference between the two types of two-dimensional representations $\left(\mathrm{CR}_{\mathrm{T}}=.083, \mathrm{p}<.01\right.$; see Figure 1$)$. The pattern revealed in Experiment 1 is thus also found here; the size of the difference between solids and their representations is greater, due perhaps to the lack of carryover effects in the between-subjects design used here.

Kendall's W for shape ranks of exponents, across modes and experiments (Experiments 1 and 2), was $.52(\mathrm{p}<.01)$; it was $.56(\mathrm{p}<.01)$ for mode ranks across shapes and experiments. Hence, there was concordance in exponent ranks across the two experiments for both modes and shapes.
Analysis of variance of log magnitude estimations revealed results similar to those of Experiment 1 . The main effects of mode $[F(2,57)=7.16]$, shape $[F(6,342)=$ $34.62]$, and volume $[F(4,228)=1726.60]$ were significant at the $.01, .001$, and .001 levels, respectively. As before, Shape by Volume $[F(24,1368)=2.40]$ and Mode by Volume $[F(8,228)=6.86]$ were significant at the .001 level. Note that the Shape by Mode interaction $[F(12,342)=1.12]$ was not significant here. Its appearance in Experiment 1 may have been due to the sensitivity of the repeated measures design or to the occurrence of sampling error. The secondorder interaction again was not significant.

Results of pairwise comparisons of log mean estimations were similar to those in Experiment 1. Differences in mode exist between the solids and the twodimensional stimuli $\left(\mathrm{CR}_{\mathrm{T}}=.088, \mathrm{p}<.01\right)$ for volumes below the standard (see Figure 1). Shape log means cluster in complex groups, but once again, in general, cylinders and tetrahedrons tend to receive the highest $\log$ means, while cubes and spheres tend to receive the lowest (see Table 3 ). Despite differences in design and new sets of subjects, the results are generally consistent with those of the first experiment.

\section{EXPERIMENT 3}

Both Experiments 1 and 2 revealed a significant, although quite small, shape effect on the estimation of apparent volume and its rate of growth. This effect, in part, involved cylinders and tetrahedrons. Both cylinders and tetrahedrons differ from the other five shape classes in possessing an elongated vertical dimension. That is, at any volume they "tower" over the five other shapes and they receive higher mean magnitude estimations; on the average, they are seen as $20 \%-30 \%$ larger than spheres or cubes. Been, Braunstein, and Piazza (1964) and Pearson (1961, 1964) reported that volume reduction was sensitive to variations in height/width ratios of solids. If two cylinders of equal volume are reduced to new, identical volume magnitudes-one cylinder, by decreasing its height, and the other cylinder, by decreasing its widththe cylinder whose height dimension is reduced appears smaller. This observation may relate to a similar effect with one-dimensional stimuli, the horizontalvertical illusion, as well as to similar effects reported with area judgments of two-dimensional figures (Anastasi, 1936; Holmberg \& Holmberg, 1969; Warren \& Pinneau, 1955). As a further test of the effect of the height/width variable, a third experiment looked at shape configuration effects within a shape class.

\section{Method}

Subjects. Twenty volunteer subjects participated. The group consisted of 17 males and 3 females. Each subject received class credit for participation. 
Apparatus. Three sets of solid cylinders were used: a control set, the same cylinders used in Experiment 1; a short set, the largest cylinder of the control set plus eight additional cylinders that retained the diameter of this largest cylinder and matched the other volumes by reduction of height only; and a tall set, the largest cylinder of the control set plus eight additional cylinders that retained this cylinder's height and matched the other volume magnitudes by reduction in base diameter (see Figure 2). As before, deviations from specified volumes were limited to under $5 \%$.

Procedure. Subjects provided magnitude estimations of apparent volume after a practice task judging length. They were first shown the standard solid sphere used previously and told to call it " 10. " Then cylinders were presented in one of 20 random orders, one order per subject. Subjects judged each of the 27 cylinders once in a single session.

\section{Results}

Best fitting power functions were derived for the data of the three sets. The functions obtained are shown in Figure 3. The nine datum points for the three cylinder sets lie along reasonably straight lines, with the exception of the lowest point for the short set, for which it appears subjects could not distinguish the lowest two volumes.

Exponents were subjected to an analysis of variance. The set factor $[F(2,38)=9.71]$ was significant $(\mathrm{p}<.001)$. Pairwise comparisons indicated that the exponent for the short set was larger than that for the control set $\left(C R_{T}=.073, p<.05\right)$ and that for the tall set $\left(C R_{T}=.093, p<.01\right)$; the control and tall sets were not different.

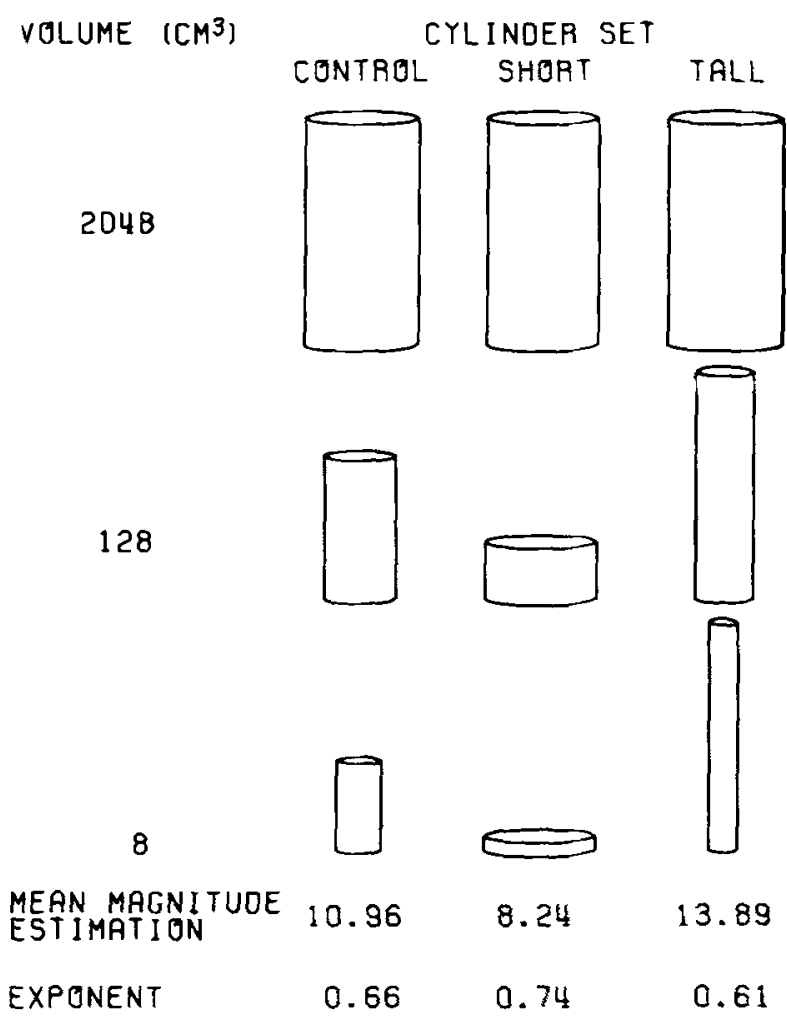

Figure 2. Stimuli, average estimates, and power function exponents for Experiment 3. (Shapes are for illustration; they are not to scale.)

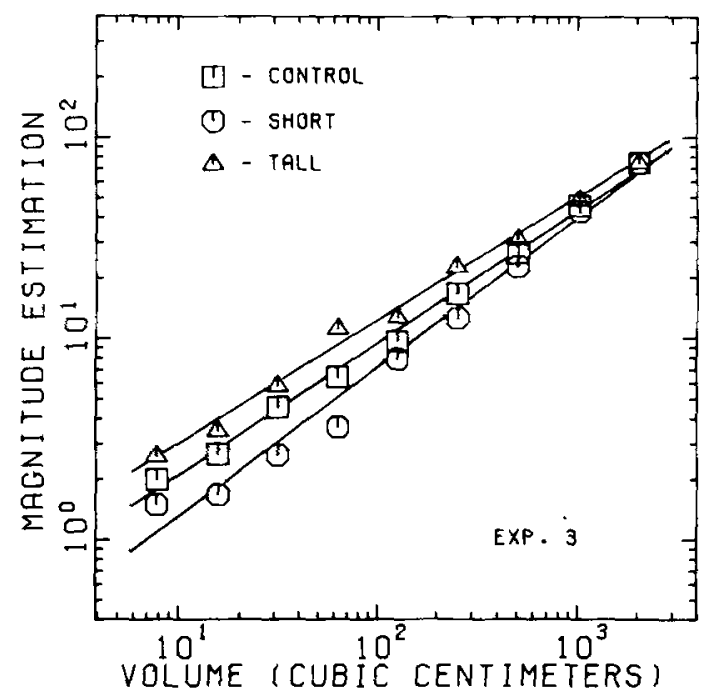

Figure 3. Geometric means of magnitude estimations from Experiment 3 for cylinders of the control set (constant height/diameter ratio-squares), the short set (height varied-octagons), and the tall set (diameter varied-triangles).

Log magnitude estimations were also subjected to an analysis of variance. Cylinder set $[\mathrm{F}(2,38)=44.48]$, volume $[F(16,152)=446.55]$, and the Set by Volume interaction $[F(16,304)=5.19]$ were all significant $(p<.001)$. When data for the largest cylinder, common to all sets, were dropped and the analysis was rerun, the interaction was still significant, showing that it was not due solely to the common cylinder.

Comparisons of mean magnitude estimations by cylinder set were carried out. At each of the nine volumes (see Figure 3), the tall set received the highest value, with control values intermediate, and short values the lowest. Generally, the statistical tests showed that, at lower volumes (below $128 \mathrm{~cm}^{3}$ ), values for the short set were less than those for the control and the tall sets, but the control and the tall sets were the same; at intermediate volumes, only the short and tall sets differed; and at higher volumes (above $256 \mathrm{~cm}^{3}$ ), there were no reliable differences $\left(C R_{T}=.014, p<.01\right)$. Overall, the mean magnitude estimation for the short set was 8.24 , for the control set, 10.96 , and for the tall set, 13.89. Note that since the sets share the common 2,048- $\mathrm{cm}^{3}$ cylinder, they also share a common datum point at that volume. As a result, the pattern of estimates across sets produces the differences in exponents (see Figure 2).

\section{DISCUSSION}

\section{The Average Exponent for Apparent Volume}

The average of the 45 exponents collected in the experiments reported here is .561 with a standard deviation of .05 . For all values available in previous reports, 37 exponents in all, the average exponent for apparent volume is .704 with a standard deviation of .08 . The average of all 82 exponents is .625 . As 
Teghtsoonian $(1959,1965)$ has contended, the exponent for judged volume is less than those reported for apparent length and apparent area.

\section{The Effect of Ocject Shape- The Vertical Elongation Effect}

There may be a basic principle that, in part, explains the effect of shape on exponents and magnitude estimations of apparent volume. One of the simplest of the common visual illusions is the horizontal-vertical illusion. This phenomenon may appear in two- and three-dimensional figures as well (Holmberg \& Holmberg, 1969; Pearson, 1964). In the present experiments, the control and tall sets, as well as the tetrahedrons, clearly "tower" over other shapes at identical volumes, whereas spheres, cubes, octahedrons, dodecahedrons, and icosahedrons are more compact. Compactness affects size judgments of two-dimensional shapes (Anastasi, 1936; Martinez \& Dawson, 1973; Smith, 1969), and the present results suggest it affects volume judgments as well, since cylinders and tetrahedrons generally appeared larger than other shapes, whereas spheres and cubes, the more geometrically compact of the solids, appeared the smallest.

The vertical elongation effect is demonstrated in Figures 2 and 3. Cylinders of the short set received the lowest log mean estimation at any volume when compared with the other two sets. This may be due to the extreme reduction of the vertical dimension of cylinders in this set. Width changes of the tall set accentuate the vertical dimension, resulting in higher estimates at each volume. These results are consistent with those of Pearson, who found that reduction in height increases apparent volume reduction more rapidly than a reduction in width. Note, however, that despite differences in the magnitude of elongation, the tall set does not appear to be significantly larger to the subjects than the control set at any volume. Perhaps, under the conditions used here, the amounts of difference in vertical elongation are not great enough to produce significance in these comparisons. Each of the tall cylinders, on the average, is judged greater than its corresponding control cylinder, and the differences in several cases approach statistical significance.

\section{The Effect of Mode}

There is a clear difference between solids and their two-dimensional representations, as to both exponents and magnitude estimations. Despite the fact that perspective drawings may provide impoverished cues to depth, judgments of these stimuli are similar to those for the photographs, in both magnitude estimations and exponents.

The effect on exponent is not so strong as Ekman and Junge originally proposed. Since they considered the exponent for solids to be 1.0 , an exponent of .79 for perspective drawings suggested a significant reduction due to a change in mode. In the present research, mode produced a much more modest exponent depression.

The source of the small yet consistent effect is not clear. Ekman and his co-workers claimed that the depression in the volume exponents for two-dimensional representations was the result of an inability of subjects to perceive volume with these stimuli. Plane figures simply provide too few depth cues, with the result that subjects base their judgments on the length of a single dimension or on area, but not on apparent volume.

The purpose of the present experiments was not to prove or disprove these hypotheses, but to determine if the mode effect actually exists or is only an artifact of comparisons made with the abnormally high exponent for solids reported by Ekman and Junge (1961). Since a significant effect does exist, even when comparisons are made against lower exponents for solids, a renewed search for the source of the mode effect seems justified.

\section{REFERENCES}

Anastasi, A. The estimation of area. Journal of General Psychology, 1936, 14, 201-225.

BAIRD, J. C. Psychophysical analysis of visual space. Oxford: Pergamon Press, 1970.

Been, R. T., Braunstein, M. L., \& Piazza, M. H. Judgment of volume reduction in distorted metal containers. Journal of Engineering Psychology, 1964, 3, 23-27.

ExMaN, G., \& JUNGE, K. Psychophysical relations in visual perception of length, area, and volume. Scandinavian Journal of Psychology, 1961, 2, 1-10.

Fejes Toth, L. Regular figures. In I. N. Sneddon, S. Ulam, \& M. Stark (Eds.), International series of monographs on pure and applied mathematics (Vol. 48). Oxford: Pergamon Press, 1964.

Frayman, B. J. The effect of object shape and mode of presentation on judgments of apparent volume. Unpublished doctoral dissertation, University of Notre Dame, 1976.

Holmberg, L., \& Holmberg, I. The perception of the area of rectangles as a function of the ratio between height and width. Psychological Research Bulletin, 1969, 9 (3), 1-6.

KEPPEL, G. Design and analysis: A researcher's handbook. Englewood Cliffs, N.J: Prentice-Hall, 1973.

Martinez, N., \& DAwson, W. E. Ranking of apparent area for different shapes of equal area. Perceptual and Motor Skills, 1973, 37, 763-770.

Moyer, R. S., Bradley, D. R., Sorensen, M. H., Whiting, J. C., \& Mansfield, D. P. Psychophysical functions for perceived and remembered size. Science, 1978, 200, 330-332.

PEARson, R. G. Judgment of volume from two-dimensional representations of complex irregular shapes. Unpublished doctoral dissertation, Carnegie Institute of Technology, 1961.

Pearson, R. G. Judgment of volume from photographs of complex shapes. Perceptual and Motor Skills, 1964, 18, 889-900.

Sмiтh, J. P. The effects of figural shape on the perception of area. Perception \& Psychophysics, 1969, 5, 49-52.

Stevens, S. S., \& Guirao, M. Loudness, reciprocality and partition scales. Journal of the Acoustical Society of America, $1962,34,1466-1471$. 
Teghtsoonian, M. The apparent size of one-, two-, and threedimensional figures. Unpublished doctoral thesis, Radcliffe College, 1959.-

Teghtsoonian, M. The judgment of size. American Journal of Psychology, 1965, 78, 392-402.

Warren, J. M., \& Pinneau, S. R. Influence of form on judgment of apparent area. Perceptual and Motor Skills, 1955, 5, 7-10.

\section{NOTES}

1. Graphical plots of these functions are available in the first author's unpublished PhD. dissertation (Frayman, 1976).

2. Detailed tables summarizing pairwise comparisons in all three experiments are available by request from the first author.

(Received for publication November 1, 1979; revision accepted October 9, 1980.) 